\title{
Osteomalacia and osteoporosis
}

\author{
D. B. MORGAN \\ Department of Clinical Investigation, University of Leeds
}

Osteomalacia and osteoporosis are still sometimes confused because both diseases lead to a deficiency of calcium which can be detected on radiographs of the skeleton.

This lack of calcium is the only feature common to the two diseases which are in all other ways easily distinguishable.

\section{Osteomalacia}

Osteomalacia will be discussed first, because it is a clearly defined disease which can be cured. Osteomalacia is the result of an imbalance between the supply of and the demand for vitamin D. The following description of the disease is based on our experience of twenty-two patients with osteomalacia after gastrectomy; there is no evidence that the features vary with the cause of the disease.

The clinical features are nearly specific; the common ones are bone pains and muscle weakness. The bone pains are generalized, often worse at night, and sometimes associated with tenderness of the bones. The bone pains have been mistakenly attributed to rheumatoid arthritis, osteoarthritis or even neurosis, and the diagnosis of osteomalacia may be delayed for years.

The muscle weakness is most obvious in the muscle around the pelvis, and a common symptom is difficulty in climbing stairs and in sitting up in bed without the aid of the arms.

The muscles may be wasted as well as weak but there is usually no evidence of neurological damage; the weakness is a symptom of a primary muscle disorder. The radiological signs of the disease may be no more than a generalized lack of calcium in the skeleton indistinguishable from that in osteoporosis. In addition, Looser's nodes are a radiological finding which is almost specific for osteomalacia. However, the nodes are not always present, and similar lesions arise in Paget's disease of bone, hyperparathyroidism and thyrotoxicosis (Camp \& McCullogh, 1941).

Looser's nodes are also known as pseudofractures because they look like fractures on the radiographs, but they are often painless.

The common and constant biochemical change in osteomalacia is an increase in the alkaline phosphatase activity in the blood (SAP); there may also be a low serum phosphorus or a low serum calcium.

Our experience with the biopsy of bone is that a large excess of uncalcified bone tissue (osteoid), which is the classic histological feature of osteomalacia, is only found in patients with the other typical features of the disease, in particular the clinical ones (Morgan et al., 1967a). Whether or not more subtle histological techniques will detect earlier stages of the disease remains to be seen.

Bone pains, muscle weakness, Looser's zones, raised SAP and low serum phosphate are the most reliable aids to the diagnosis of osteomalacia, and approximately in that order.

The interpretation of a raised SAP is difficult because the 'normal values' for SAP are not clearly defined. The particular problem is that the SAP increases with age in the normal population (Hobson \& Jordan 1959; Klaasen \& Sierstema, 1964); values of 16 or even 25 KA units/ $100 \mathrm{ml}$ of serum are not uncommon in well persons over 60 years of age. Even if the SAP is above these values it may indicate some other disease of bone, for example Paget's disease, or even chronic liver disease, rather than osteomalacia.

A raised SAP together with generalized bone pains, could be the result of Paget's disease of bone rather than osteomalacia and it may be impossible to distinguish these two diseases even on biopsy of the bone (Paterson, Woods \& Morgan, 1968). That Paget's disease is radiologically detectable is no proof that it is the cause of the symptoms and the presence of Paget's disease which is common does not exclude osteomalacia which is rare.

For these reasons we have come to regard a response to vitamin $D$ as the essential and final diagnostic criterion of osteomalacia.

Historically, osteomalacia was recognized as the adult counterpart of rickets in children, that is to say osteomalacia was the result of a simple deficiency of vitamin D usually the result of a poor dietary intake of the vitamin. Osteomalacia thus defined still occurs (Gough, Lloyd \& Wills, 
1964 ; Andersson et al., 1966; Chalmers et al., 1967). This condition can usually be cured with 3000 i.u. of vitamin D a day. The daily requirement for the vitamin in the diet is probably of the order of 100 i.u. $(2.5 \mu \mathrm{g})$ in adults. Unfortunately, the term osteomalacia has been applied to those conditions which simulate nutritional osteomalacia in their clinical, biochemical, and histological signs, but which are not the result of a simple deficiency of vitamin $D$.

These 'osteomalacia-like' diseases fall into several groups according to the dose of vitamin $D$ required to achieve some response and the fundamental abnormality in the disease.

At one extreme is the group of diseases which includes familial hypophosphataemia. These diseases are not cured by vitamin $\mathrm{D}$ however much of the vitamin is given.

The osteomalacia of chronic glomerular failure heals with vitamin $D$ but only in very large doses $(150,000$ i.u. a day or more). Stanbury \& Lumb (1966), therefore, suggested that this condition is the result of a resistance to the action of the vitamin and thus an increased demand for it. This demand is not met by the amount of the vitamin in the diet.

The position within this spectrum of the osteomalacia of intestinal disease depends on the form of the latter. Osteomalacia after resection of the distal intestine or in association with regional ileitis is usually the result of a simple deficiency of vitamin $D$, perhaps due to a failure to absorb the vitamin. On the other hand, the osteomalacia associated with malabsorption due to gluten sensitivity may be partly the result of a resistance to the vitamin (Nassim et al., 1959).

In order to establish the nature of the osteomalacia after gastrectomy we gave patients with the clinical and biochemical features of the disease, small doses of the vitamin of the order of the physiological requirement for the vitamin; in this case 1000 i.u. $(25 \mu \mathrm{g})$ weekly by intramuscular injection (Morgan et al., 1965b). The patients responded clinically, biochemically, radiologically and histologically to this treatment. Such a response not only established the presence of osteomalacia in these patients but also demonstrated that the osteomalacia was the result of a simple deficiency of vitamin $D$. These small doses were chosen to establish the presence of a deficiency of the vitamin; we would not of course suggest that they represent the most efficient treatment for the disease.

\section{The treatment of osteomalacia}

The dose of vitamin $D$ which is effective clearly depends on the cause of the osteomalacia.
For the osteomalacia after gastrectomy we have given one dose of $10 \mathrm{mg}$ of vitamin $D_{2}$ by intramuscular injection. This dose will relieve the bone pains, heal the Looser's nodes, and the SAP and serum phosphorus and calcium return to normal. The SAP may rise after the vitamin $D$, before it begins to fall towards normal values. The increase in the serum phosphorus may be the most dramatic and reliable measure of a response to vitamin $D$ but only if the measurements are made in the fasting patient. The frequency at which further injections are required varies much from one patient to another. We have controlled the frequency of injection by the symptoms and the SAP. Some of our patients required the injections every 4 months, but one of our patients with severe osteomalacia, who had only one injection almost 3 years ago, became symptom-free in a few months and has remained well since. A safe prescription and a liberal one would be an injection of $1 \mathrm{mg}$ of vitamin $\mathrm{D}_{2}$ every month.

Once the diagnosis is established it is essential to supplement the diet with calcium, otherwise the bone may heal at the expense of the calcified skeleton, and the osteomalacia may become osteoporosis.

Treatment should be continued for at least 2 years, even if the symptoms have disappeared earlier and the biochemistry has returned to normal.

A recurrence of the disease can probably be prevented by 1000 i.u. of vitamin $D$ by mouth every day for life. Supplementation of the diet with calcium is not necessary after the bones have healed.

\section{The cause of the vitamin deficiency}

We get our vitamin $D$ from the diet by making the vitamin in the skin through a trapping of the energy in the ultraviolet light from the sun.

Taking into account the many factors which diminish the amount of ultraviolet light that reaches us, the amount of the vitamin we make in the skin is probably small and may be insignificant in relation to our need for the vitamin.

The deficiency of vitamin $D$ in patients with osteomalacia after gastrectomy is, therefore, the result of too small an intake of the vitamin or a failure to absorb the vitamin or both.

There is no simple explanation of the deficiency which applies to all patients with osteomalacia after gastrectomy, and it seems likely that a deficiency only arises when all the factors which can diminish the supply of the vitamin coincide in one patient. 
The frequency of osteomalacia

In a survey of 1228 patients who had had a stomach operation for peptic ulcer in York since 1940 we saw only six patients with osteomalacia as we have defined it (Morgan et al., 1965a). None of these patients had had a vagotomy and drainage procedure. We have since seen four more patients with the disease so that the frequency of osteomalacia after Polya-type gastrectomy was only about $1 \%$ in men and $4 \%$ in women.

The disease was usually first obvious to the patient $8-12$ years after operation. This is also true of the other patients described in the literature and suggests that the patients who will later develop osteomalacia are destined to do so from about the time of operation.

There have been various opinions concerning the existence and frequency of 'sub-clinical osteomalacia' after stomach operations.

We have never denied the existence of a stage of osteomalacia where the biochemical and histological changes are minimal but yet respond to vitamin $D$. We have only one patient who might have been at this stage of the disease. We, therefore, conclude that 'subclinical osteomalacia' soon progresses to typical osteomalacia and would only be detected by chance. Thompson and his colleagues (1966) suggested that subclinical osteomalacia is a common disease after gastrectomy. If this is so then in our experience sub-clinical osteomalacia only rarely progresses to obvious osteomalacia.

\section{Osteoporosis}

The term osteoporosis has difficult connotations to the histologist, the radiologist and the clinician; the term now has little practical significance. Osteoporosis, however defined, is common in persons who have not had a stomach operation. It is the definition of osteoporosis and the clinical problem of osteoporosis in the general population which I will discuss following $\mathrm{Dr}$ Pulvertaft's paper on osteoporosis after stomach operations.

Osteoporosis was originally defined as the condition of too little bone when the bone which remained was fully calcified. Thus histological examination of the bone should have been essential to establish the diagnosis.

The loss of bone may be detected on radiographs as a lack of calcium but it is difficult to interpret the radiographs subjectively, and even if lack of calcium is obvious it could be due to osteomalacia or to osteoporosis.

In some cases the loss of bone is associated with deformities or fractures of the bones. These deformitities and fractures have been attributed to the loss of bone together with a deterioration in the quality of the bone tissue. But in the case of osteoporosis in the elderly there is no great change in the quality of the bone and the failure of the bones is probably explained by the loss of bone tissue.

In clinical practice, the diagnosis of osteoporosis is applied in two distinct circumstances. The first is when the bones are found to be 'thin' on radiographs of the spine, taken for some unrelated symptoms. The diagnosis in the second group is based on the presence of complaints which are attributed to a loss of bone subsequently detected on radiographs. Thus backache and rarely spine shortening and deformity are attributed to vertebral collapse due to loss of bone from the vertebrae. Fractures of the long bones are attributed to a loss of bone tissue from the long bones.

The question in dispute is whether there is a distinct disease (osteoporosis) with a loss of much of the bone tissue, which can be detected by the histologist and the radiologist as a discrete condition and which gives rise to symptoms which take the patient to the physician or the orthopedic surgeon.

The reason for the dispute is that it is now realized that there is a loss of bone tissue in all persons as they age and it is not yet agreed whether this 'physiological' loss of bone is sufficient to account for the clinical problem of osteoporosis.

Loss of bone tissue with age affects the peripheral skeleton as well as the axial skeleton and cortical bone as well as trabecular bone. Symptoms which may be attributed to the loss of bone are most common in the spine and routine radiography would suggest that most bone is lost from the spine. However, all the measurements of the amount of bone lost with age show that as much is lost from the peripheral skeleton as from the spine.

Although subjective assessment of routine radiography of the spine is potentially misleading in the individual, the technique has some value if applied to large numbers of individuals so that the errors are randomly distributed. Smith \& Rizek (1966) assessed the radiographs of the vertebrae of 1200 normal women, and their results show clearly that there is a steady loss of bone from the vertebrae after the age of 45 .

The factors which reduce the reliability of the routine radiography of the spine are the unavoidable variations in exposure and development of the radiographs and the large and variable amount of soft tissue around the spine. E 
Several techniques have been described which give an objective assessment of the amount of bone tissue in the vertebrae (Nordin et al., 1962; Oeser \& Krokowski, 1963 ; Hurxthal et al., 1964). However, these techniques lack precision and they have given most information in epidemiological studies. All three studies show a loss of bone with age; Oeser and Krokowski who were the only ones to study the bone loss in men and in women found that the loss of bone started earlier and was more rapid in women than men.

There are many more data for the changes in the peripheral bone with age, since there is less soft tissue to complicate densitometric techniques and in any case the thickness of the cortical bone is a simple measure of the amount of bone in the periphery.

All these studies show a loss of bone with age which starts earlier and is more rapid in women than men (Meema, 1962; Garn, Rhohman \& Nolan, 1964 ; Nordin, McGregor \& Smith, 1966; Smith \& Rizek, 1966; Morgan et al., $1967 b)$. Women lose, on the average, $40-50 \%$ of their bone tissue between the fifth and the ninth decades. The obvious suggestion is that the loss of bone in women follows and may be the result of the menopause. Meema, Bunker \& Meema (1965) have obtained some direct evidence to support this suggestion.

All, or nearly all, persons lose bone as they age, and there is no evidence of a group of persons who lose more bone than the others and might, therefore, be called 'osteoporotic'. For these reasons the definition of osteoporosis as a loss of bone tissue is of no value in clinical practice.

It is, therefore, necessary to restrict the definition in some way. The obvious suggestion is to confine the term osteoporosis to those persons where the loss of bone tissue has allowed structural failure.

Biconcavity of the vertebral bodies and wedge fractures of the vertebrae have both been taken as evidence of structural failure in the spine. True gross biconcavity of the vertebrae as opposed to apparent biconcavity on radiographs is rare even in the elderly, probably because gross biconcavity will only arise if the intervertebral discs retain their turgidity and the intervertebral discs tend to become less turgid in the elderly. There is no relation between gross biconcavity and the amount of bone in the vertebrae (Caldwell, 1962 ; Virtama, Gastrin \& Telkka, 1962). Attempts to detect lesser degrees of biconcavity have failed (Nordin et al., 1966) or have been abandoned because of lack of precision (Smith \& Rizek, 1966). On the other hand the frequency of wedge fractures of the spine is greatest in the vertebrae containing least bone (Smith \& Rizek, 1966).

Structural failure in the form of wedge fractures of the spine and fractures of the long bones increases in frequency with age and is more common in women than men.

The studies of Meema \& Meema (1963) and Nordin (1966) show that persons with structural failure come from among the persons with least bone, but there is no evidence that persons with structural failure form a distinct group with less bone than is 'normal' for persons of the same age and sex. Thus even the persons with structural failure when considered according to the amount of bone in the skeleton do not form a distinct group with less bone than normal.

The most difficult problem is the relation between structural failure in the spine and symptoms. The expected symptom is backache and specific characteristics of the backache of spinal osteoporosis have been suggested (Dent \& Watson, 1966). However, backache is no more common in the elderly than the young or in women than in men (Nordin et al., 1966 ; Morgan \& Pulvertaft, unpublished observations).

Also there is no relation between wedge fractures and backache even in elderly persons (Gitman, Kamholtz \& Levin, 1958 ; Smith, Eyler \& Mellinger, 1960). Thus the apparent association between backache and fracture of the spine may be explained by the high frequency of both phenomena which will be commonly associated although not necessarily casually related.

There are a few patients who have less bone than is 'normal' for their age and sex; these patients are often young men. The fundamental abnormality in this condition is not known, but it seems to be different from the disturbance which leads to the loss of bone which accompanies aging (Hioco, Miraret \& Bordier, 1964).

Cushing's syndrome, thyrotoxicosis and osteogenesis imperfecta should be considered as causes of 'thin bones' at all ages, particularly in the young ; 'thin bones' may be the only sign of Cushing's syndrome (Fourman \& Thomas, 1964). However, all these causes of 'thin bones' are rare compared with the frequency of 'thin bones' in the elderly.

As to the treatment of the loss of bone which accompanies ageing it is clear that the loss can at best be stopped, never corrected. Treatment with oestrogens and calcium supplements both have strong advocates but it is as yet impossible to choose between these two means of arresting the loss of bone.

The therapy of the future may be an attempt to prevent the loss of bone by treating some or 
all the patients prophylactically; women from the age of 40 years, men from the age of about 60 years.

\section{Conclusions}

Osteomalacia is a metabolic bone disease with characteristic biochemical, radiological and histological features. Unfortunately not all of these features need be present in the individual and few of the features are specific for osteomalacia. For these reasons we believe that a response to vitamin $D$ is the essential diagnostic criterion of the disease. Osteomalacia is an unusual complication of gastric surgery. When it does happen it is the result of a simple deficiency of vitamin $\mathrm{D}$ and treatment with small doses of the vitamin brings early relief of symptoms and heals the bones.

There is no evidence of a distinct disorder of 'osteoporosis' in the elderly other than as one extreme of the loss of bone which occurs in all persons as they age. The only exceptions are the rare identifiable causes of 'thin bones' such as Cushing's syndrome and thyrotoxicosis.

The loss of bone with age is sometimes associated with fracture of the spine and occasionally with fracture of the long bones. However, there is little relation between fracture of the spine and backache, and the syndrome of backache and fracture of the spine may often be a chance association of two common happenings in the elderly.

\section{References}

Anderson, I., Campbell, A.E.R., DunN, A. \& Runciman, J.B.M.. (1966) Osteomalacia in elderly women. Scot. med. J. 11, 429.

Caldwell, R.A. (1962) Observations on the incidence, aetiology and pathology of senile osteoporosis. J. clin. Path. 15, 421.

CAmp, J.D. \& MCCullogh, J.A.L. (1941) Pseudofractures in diseases affecting the skeletal system. Radiology. 36, 651 .

Chalmers, J., Conacher, W.D.H., Gardner, D.L. \& ScotT, P.J. (1967) Osteomalacia-a common disease in elderly women. J. Bone Jt Surg. 498, 403.

Dent, C.E. \& Watson, L. (1966) Osteoporosis. Postgrad. med. J. Suppl. (October).

Fourman, P. \& Thomas, J.P. (1964) L'Ostéoporose (Ed. by D. J. Hioco), p. 191. Masson et cie, Paris.

Garn, M.S., RohmanN, C.O. \& Nolan, P. (1964) Relations of Development and Ageing (Ed. by J. E. Birren). Thomas, Springfield, Illinois.

Gitman, L., Kamholtz, T. \& Levin, J. (1958) Osteoporosis in the aged: radiographic survey with clinical and biochemical correlations. J. Geront, 13, 43.
Gough, K.R., Lloyd, O.L. \& Wills, M.R. (1964). Nutritional osteomalacia. Lancet, ii, 1261.

Hioco, D., Miravet, L. \& Bordier, P. (1964) Bone and Tooth (Ed. by H. J. J. Blackwood). Pergamon Press, London.

Hobson, W. \& Jordan, A. (1959) A study of serum alkaline phosphatase levels in old people living at home. J. Geront. 14, 292.

HurXthal, L.M., Dotter, W.E., Baylink, D.J. \& Vose, G.P. (1964). Two new methods for the study of osteoporosis and other metabolic bone diseases. Lahey Clin. Bull. 13, 155.

KlaAsen, C.H.L. \& Sierstsema, L.H. (1964) De invloed van de leeftijd op de alkalische-fosfatase waarde in het serum. Ned. Tijdkr Geneesk. 108, 1433.

MEEMA, H.E. (1962) The occurrence of cortical bone atrophy in old age and in osteoporosis. J. Can. Ass. Radiol. 13, 27.

Meema, H.E. \& Meema, S. (1963) Measurable roentgenologic changes in some peripheral bones in senile osteoporosis. J. Amer. ger. Soc. 11, 1170.

Meema, H.E., Bunker, M.L. \& Meema, S. (1965) Loss of compact bone due to menopause. Obstet. Gynaec. 26, 333.

Morgan, D.B., Paterson, C.R., Woods, C.G., Pulvertaft, C.N. \& Fourman, P. (1965a) Search for osteomalacia in 1228 patients after gastrectomy and other operations on stomach. Lancet, ii, 1085.

Morgan, D.B., Paterson, C.R., Woods, C.G., PulverTAFT, C.N. \& Fourman, P. (1965b) Osteomalacia after gastrectomy. Lancet, ii, 1089.

Morgan, D.B., Lever, J.V., Paterson, C.R., Woods, C.G., Pulvertaft, C.N. \& Fourman, P. (1967a) Osteomalacia (Ed. by D. J. Hioco). Masson et cie, Paris.

Morgan, D.B., Spiers, F.W., Pulvertaft, C.N. \& FourMAN, P. (1967b) The amount of bone in the metacarpal and the phalanx according to age and sex. Clin. Radiol. 18, 101.

Nassim, J., Saville, P., Cook, P., Milligan, G. (1959) The effects of vitamin $D$ and gluten-free diet in idiopathic steatorrhoea. Quart. J. Med. 28, 141.

Nordin, B.E.C. (1966) Medicine in Old Age (Ed. by J. N. Agate), p. 5. Pitman Medical.

Nordin, B.E.C., Barnett, E., McGregor, J. \& Nisbet, J. (1962) Lumbar spine densitometry. Brit. med. J. i, 1793.

Nordin, B.E.C., McGregor, J. \& Smith, D.A. (1966) The incidence of osteoporosis in elderly women: its relation to age and the menopause. Quart.J. Med. 35, 25.

OESER, H. \& KROKowsKi, E. (1963) Quantitative analysis of inorganic substances in the body. Brit. J. Radiol. 36, 274.

Paterson, C.R., Woods, C.G. \& Morgan, D.B. (1968) Osteoid in metabolic bone disease. J. Path. Bact. $95,449$.

Smith, R.W., Eyler, W.R. \& Mellinger, R.C. (1960) On the incidence of senile osteoporosis. Ann. intern. Med. 52, 773.

SMith, R.W. \& RizeK, J. (1966) Epidemiologic studies of osteoporosis in women of Puerto Rico and south eastern Michigan with special reference to age, race, national origin and to other related or associated findings. Clin. orthop. 45,31 .

Stanbury, S.W. \& Lumb, G.A. (1966) Parathyroid function in chronic renal failure. Quart. J. Med. 35, 1.

Thompson, G.R., Neale, G., Watts, J.M. \& Booth, C.C. (1966) Detection of vitamin D deficiency after partial gastrectomy. Lancet, i, 623.

Virtama, P., Gastrin, G. \& Telkka, A. (1962) Biconcavity of the vertebrae as an estimate of their bone density. Clin. Radiol. 13, 128. 